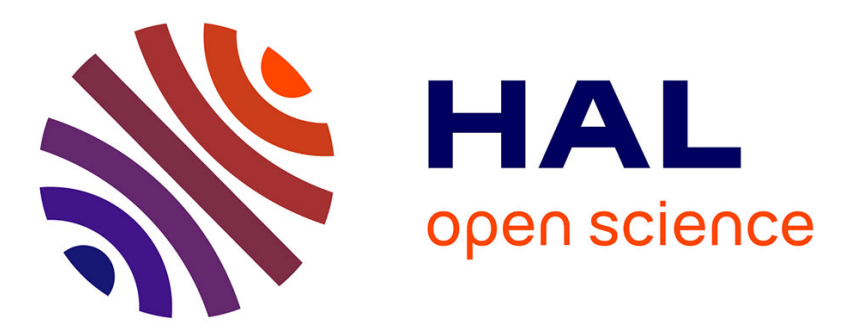

\title{
Comparative Study of Forced Oscillators for the Adaptive Generation of Rhythmic Movements in Robot Controllers
}

\author{
Melanie Jouaiti, Patrick Henaff
}

\section{- To cite this version:}

Melanie Jouaiti, Patrick Henaff. Comparative Study of Forced Oscillators for the Adaptive Generation of Rhythmic Movements in Robot Controllers. Biological Cybernetics (Modeling), 2019, 113 (5-6), pp.547-560. 10.1007/s00422-019-00807-8 . hal-02291575

\section{HAL Id: hal-02291575 \\ https://hal.science/hal-02291575}

Submitted on 19 Sep 2019

HAL is a multi-disciplinary open access archive for the deposit and dissemination of scientific research documents, whether they are published or not. The documents may come from teaching and research institutions in France or abroad, or from public or private research centers.
L'archive ouverte pluridisciplinaire $\mathbf{H A L}$, est destinée au dépôt et à la diffusion de documents scientifiques de niveau recherche, publiés ou non, émanant des établissements d'enseignement et de recherche français ou étrangers, des laboratoires publics ou privés. 


\title{
Comparative Study of Forced Oscillators for the Adaptive Generation of Rhythmic Movements in Robot Controllers
}

\author{
Melanie Jouaiti • Patrick Hénaff
}

Received: date / Accepted: date

\begin{abstract}
The interest of Central Pattern Generators (CPGs) in robot motor coordination is universally recognized so much so that a lot of possibilities on different scales of modeling are nowadays available. While each method obviously has its advantages and drawbacks, some could be more suitable for human-robot interactions.

In this paper, we compare three oscillator models: Matsuoka, Hopf and Rowat-Selverston models. These models are integrated to a control architecture for a robotic arm and evaluated in simulation during a simplified handshaking interaction which involves constrained rhythmic movements. Furthermore, Hebbian plasticity mechanisms are integrated to the Hopf and Rowat-Selverston models which can incorporate such mechanisms, contrary to the Matsuoka. Results show that the Matsuoka oscillator is subpar in all aspects and for the two others, that plasticity improves synchronization and leads to a significant decrease of the power consumption.
\end{abstract}

Keywords Oscillator · synchronization · rhythmic movements $\cdot$ robot controller

\section{Introduction}

Central Pattern Generators (CPGs) are biological structures found in the central nervous system of vertebrates or in some ganglia of invertebrates. CPGs

Melanie Jouaiti

Université de Lorraine, CNRS, LORIA, F-54000 Nancy,

France

E-mail: melanie.jouaiti@loria.fr

Patrick Hénaff

Université de Lorraine, CNRS, LORIA, F-54000 Nancy, France can generate a rhythmic signal even when the input signal is not rhythmic, modulated by afferent sensory feedbacks. While their implication in upper limb movements is strongly suspected (Schaal, 2006; Zehr et al, 2004), their role in locomotion has been recognized and widely studied. Mesoscopic CPGs are usually based on a pair of half-center neurons (Grillner and Wallen, 1985), controlling the extensor and flexor muscles. CPGs have several interpretations which differ according to the level of bio-inspiration (Ijspeert, 2008; Yu et al, 2014). Biologists usually present CPGs as complex structures which encompass sensory neurons, motor neurons and interneurons and receive sensory feedback (Rybak et al, 2006; Cattaert and Le Ray, 2001). However, in computational neuroscience, some aspects tend to not be taken into account for simplicity's sake. While some studies endeavour to be biologically accurate (Nassour et al, 2019; Taga et al, 1991; Manoonpong et al, 2008), others present simplified interpretations (Mori et al, 2004; Wu and Ma, 2010).

The interest of using CPGs in robotics is nowadays widely recognized, so much so that a great variety of possibilities has been proposed (see Yu et al (2014); Ijspeert (2008) for reviews). The term CPG refers to a network of coupled oscillators. Non-linear models of CPGs, composed of relaxation oscillators, can be entrained by an oscillatory input or with a coupled CPG if the coupling is strong enough or if the input frequency is close enough to the intrinsic frequency of the oscillator, thus ensuring coordination. Even though CPGs have mostly been used for robotic locomotion (Taga, 1995; Shan and Nagashima, 2002; Ayers, 2004; Kamimura et al, 2005; Arena et al, 2006; He et al, 2006; Pelc et al, 2008; Sprowitz et al, 2010; Liu et al, 2011; Pinto et al, 2012; Wang et al, 2013), see Ijspeert (2008) for a review, 
some studies explore how CPGs affect upper limb control as well (Williamson, 1998; Yang et al, 2010). The strength of CPGs resides in their self-synchronization ability, their oscillating stability despite perturbation and the variety of behaviors they can generate. Besides, plasticity mechanisms can be integrated, thus making the CPG even more robust and versatile. This is actually essential to have adaptive robot controllers.

In social, collaborative robotics, human-robot interactions are paramount. If humans are to be comfortable interacting with robots, robots have to behave in a coherent and adaptive way, i.e. their response has to be suited to the partner and the social context. Human interactions entail a lot of rhythmic non-verbal communication (waving, handshaking, walking) and it has been observed that humans learn from these interactions and adapt to their partner, thus leading to interpersonal synchronization and motor coordination and to a greater engagement in the interaction. It is hence important for robots to be able to replicate that. We consider the case of handshaking which is a highly social act responsible for the creation of conscious and unconscious links between the interaction partners. Despite its apparent triviality, it involves complex tasks from a neuroscience and robotics point of view, one of them being the emergence of synchronization and phase-locking observed during the act. Kasuga and Hashimoto (2005) introduced a framework for human-robot handshaking using neural oscillators. They were able to control how passive or active the robot handshake was and modulate the human perception of the response.

A great variety of oscillators can be found in the literature at different modeling scales; amongst the most popular, to name a few: microscopic (Hodgkin and Huxley, 1952) which models single neuron; mesoscopic (Rowat and Selverston, 1993; Matsuoka, 1985) which models populations of neurons, taking biological mechanisms into account and macroscopic (Hopf, 1942) which also models populations of neurons but with no bio-inspiration. To determine which oscillator is actually more suited to a given purpose and why, our contribution consists in comparing three oscillator models in the same control architecture (Kasuga and Hashimoto, 2005) and in explaining the differences observed. A similar endeavour was undertaken by Collins and Richmond (1994) who compared three different neuronal oscillator models (Stein, Van der Pol and Fitzhugh Nagumo) and two different coupling schemes for locomotion. They studied the ability of each oscillator to produce the walking, trotting, and bounding gaits, as well as the possibility to switch between multiple gait patterns. The originality of this paper resides in comparing three oscillators by evaluating them on their entrainment abilities, power consumption with and without plasticity mechanisms in the same handshaking task, which, to our knowledge, has never been done before.

This paper is organized as follows. First, we present the three oscillator models studied: Matsuoka, Hopf and Rowat-Selvertson. They were chosen because Matsuoka is a neural oscillator, Hopf a non-linear oscillator and they are both the most popular choices in robotics. Besides, we believe Rowat-Selverston to be a compromise between the two alternatives, being a non-linear oscillator inspired by Van der Pol but it also encompasses biological inspiration, making it a neural oscillator as well. Then, in section III, we compare the three oscillators, in one set of experiments on their entrainment range and in another set of experiments with their synchronization capacity. Then we integrate plasticity mechanisms to Hopf and Rowat-Selverston and evaluate the energetic cost. Finally, in section IV, we discuss our results and future prospects.

\section{Material and Method}

\subsection{Oscillating Neuron Models}

\subsubsection{Matsuoka neuron}

Introduced in 1985, the Matsuoka model (Matsuoka, 1985 ) is undoubtedly the most well-known and employed neural oscillator. In the original paper, a single neuron defined by two differential equations was studied, as well as its behaviour in networks of $n$ coupled neurons. Since Taga et al (1991), it has become customary to couple two neurons in CPGs. While this model has mostly been used for robotic biped locomotion (Liu et al, 2006, 2007, 2008; Panwart and Kumar, 2012; Liu et al, 2012; Al-Busaidi et al, 2012), some original works applied it to achieve human-robot handshaking (Kasuga and Hashimoto, 2005), a chewing robot (Xu et al, 2009) or traffic lights regulation (Fang et al, 2013). A Matsuoka neuron is a mesoscopic model defined by the following equations:

$$
\begin{aligned}
\dot{x} & =\frac{1}{T}(-x-b v+c+\epsilon F) \\
\dot{v} & =\frac{1}{\tau}(-v+y) \\
y & =\max (x, 0)
\end{aligned}
$$

where $F$ represents the input signal, $T$ and $\tau$ are time constants and $b$ the self-inhibition (or neuron fatigue), $y$ is the cell output and $c$ the excitatory tonic input. 


\subsubsection{Hopf oscillator}

The non-linear Hopf oscillator (Hopf, 1942) is particularly popular in robotics. It has been widely applied to robot locomotion ( $\mathrm{Li}$ et al, 2013; Matos and Santos, 2010; Ijspeert, 2004; Brambilla et al, 2006; Fuente et al, 2013; Buchli and Ijspeert, 2008; Righetti and Ijspeert, 2006, 2008) and robot swimming (Seo et al, 2010; Hu et al, 2011; Zhou and Low, 2012; Hu et al, 2014); but also to robot hopping (Buchli et al, 2005, 2006), drumming (Degallier et al, 2006), crawling, reaching (Degallier et al, 2008), the flight of a robotic bat (Chung and Dorothy, 2010).

The Hopf oscillator can be considered as a macroscopic model of neural oscillator structures, so it does not have the half center structure usually present in neural oscillators. The equations for a Hopf cell are defined as follows:

$\dot{x}=\left(\mu-\left(x^{2}+y^{2}\right)\right) x-\theta y+\epsilon F$

$\dot{y}=\left(\mu-\left(x^{2}+y^{2}\right)\right) y+\theta x$

with $F$ the input signal and $y$ the neuron output. For $\mu<0.5$, oscillations are damped. $\mu$ determines the output amplitude which can be influenced by $\epsilon$ the input gain. $\theta$ determines the intrinsic frequency of the oscillator $(\omega \approx 0.155 \cdot \theta)$.

\subsubsection{Rowat-Selverston Neuron}

The Rowat-Selverston neuron (Rowat and Selverston, 1993) is able to produce discrete and rhythmic activities depending of two parameters like shown in Jouaiti and Henaff (2018); Nassour et al (2014). Properties of the Van der Pol can be applied to it since it is a generalized Van der Pol oscillator, notably the dynamic Hebbian learning of frequency introduced by Righetti et al (2006), as demonstrated in Jouaiti et al (2018). This model has been underused to this day, only few studies employ it (e.g. (Jouaiti et al, 2018; Arikan and Irfanoglu, 2011; Nassour et al, 2014)).

$$
\begin{aligned}
\dot{V}= & y+\epsilon F \\
\dot{y}= & \frac{1}{\tau_{m}}\left(\sigma_{f}-\frac{\tau_{m}}{\tau_{s}}-1-\sigma_{f} \tanh ^{2}\left(\frac{\sigma_{f}}{A_{f}} V\right)\right) y \\
& -\frac{1+\sigma_{s}}{\tau_{s} \tau_{m}} V+\frac{A_{f}}{\tau_{s} \tau_{m}} \tanh \left(\frac{\sigma_{f}}{A_{f}} V\right)
\end{aligned}
$$

with $F$ the input signal and $y$ the neuron output, $V$ the cellular membrane potential, $q$ the slow current, $\tau_{m}$ the time constant of the cellular membrane, $\tau_{s}$ is the time constant of slow current activation $\left(\tau_{m} \ll \tau_{s}\right), \sigma_{s}$ and $\sigma_{f}$ represent respectively the conductance of slow and fast currents, $A_{f}$ influences the amplitude of $V$.

\subsubsection{Mathematical Plasticity Modeling}

Righetti et al (2006) presented a frequency adaptation rule for the Hopf oscillator. This rule allows the oscillator to learn the input frequency and truly adapt to the input signal. Besides, when the interaction stops, the system retains the learning and remains at the learned frequency:

$\dot{\theta}=-\eta \frac{y}{\sqrt{x^{2}+y^{2}}} F$

With $\eta$ the learning step.

For the Rowat-Selverston cells, the plasticity mechanisms previously introduced in Jouaiti et al (2018) are employed: frequency learning, amplitude learning and synaptic gain learning:

$$
\begin{aligned}
\dot{\sigma}_{s} & =2 \epsilon F \sqrt{\tau_{m} \tau_{s}\left(1+\sigma_{s}-\sigma_{f}\right)} \cdot \frac{y}{\sqrt{V^{2}+y^{2}}} \\
\dot{A}_{f} & =-\mu\left(\left(\frac{\nu \sigma_{f} V}{A_{f}}\right)^{2}-F^{2}\right) \\
\dot{\epsilon} & =\lambda \tanh ^{2}(\xi F)\left(1-(\epsilon F)^{2}\right)
\end{aligned}
$$

with $\mu$ and $\lambda$ learning steps, $\nu$ a gain modulating the output amplitude and $\xi$ a gain ensuring that $\tanh ^{2}(\xi F)$ is 0 when no input is applied and 1 otherwise.

For the Matsuoka model, one could modulate the global output as done in Taga et al (1991) or change the time constants (de Rugy et al, 2003). We will present the latter option. This method consists in comparing the oscillator period $P_{r}$ and the target period $P_{t}$ and if this difference exceeds a given threshold $\delta$, the following adaptation equation is enabled:

$$
\begin{aligned}
\tau & =c_{1} \cdot P_{t} \\
T & =c_{2} \cdot P_{t}
\end{aligned}
$$

with $c_{1}$ and $c_{2}$ two empirically determined constants and $P_{t}$ the target period, i.e. the period of the input.

\subsubsection{Parameter Tuning}

The intrinsic properties of each oscillator model can be modulated thanks to the various parameters available. Properties of interest are the intrinsic frequency and amplitude of the oscillations. 
For the Matsuoka model, the amplitude is determined by $c$, though this is merely an offset so the amplitude really does not change. For Hopf and RowatSelverston, $\mu$ and $A_{f}$ determine the amplitude respectively but it can be further influenced by the input amplitude $\epsilon F$. Note though that setting the amplitude of Hopf is rather troublesome and some output amplitudes are just impossible to set, Rowat-Selverston is more straightforward because the amplitude is proportional to $A_{f}$.

In the Matsuoka model, four parameters are necessary to set the intrinsic frequency $\left(\omega=\frac{1}{\tau} \sqrt{\frac{(T+\tau) b}{T \cdot W}-1}\right)$ and two conditions have to be met in order to obtain oscillations $(W>1+T / \tau$ and $b>W+1$ ) (see Matsuoka (2011) for details). Note also that Matsuoka synchronizes better with an input signal when it is unable to oscillate by itself. For Hopf, if $\mu<0.5$, the oscillations are damped; the intrinsic frequency depends solely on $\theta$. Finally, the Rowat-Selverston model produces oscillations if $\sigma_{f}>1+\tau_{m} / \tau_{s}$. The intrinsic frequency is dependent on $\tau_{m}, \tau_{s}, \sigma_{s}, W$. While the Rowat-Selverston model seems as difficult to control as the Matsuoka model, it really is not so. Setting a particular intrinsic frequency is, in both cases, a complicated endeavour but putting the oscillator in conditions such that it can adapt to a wide range of frequencies is actually fairly easy with Rowat-Selverston. Indeed, by choosing wisely $\tau_{m}$ and $\tau_{s}$, the oscillator can cover the desired frequency range quite easily, even if it is quite wide. Then the value of $\sigma_{s}$ hardly matters, thanks to the learning rule. On the contrary, in order to change the frequency range of the Matsuoka model, the time constants have to be changed since they're only able to cover a small range. In biological systems, the membrane time constants are set and cannot be modulated so this kind of plasticity is not biologically sound.

\subsection{Implementing the CPG controller for a robot}

\subsubsection{Coupling the oscillatory neurons}

From now on, we consider two coupled oscillators controlling the flexor and the extensor muscles in reference to the half center structure introduced by Rybak et al (2006). However, in robotics, this is hardly applicable as such since robots have a single joint in place for the flexor and extensor parts. It is then common practice to subtract both commands to obtain the output (see Figure 1).

In all equations, the term in $W$ models the mutual inhibition between the extensor and the flexor cells.

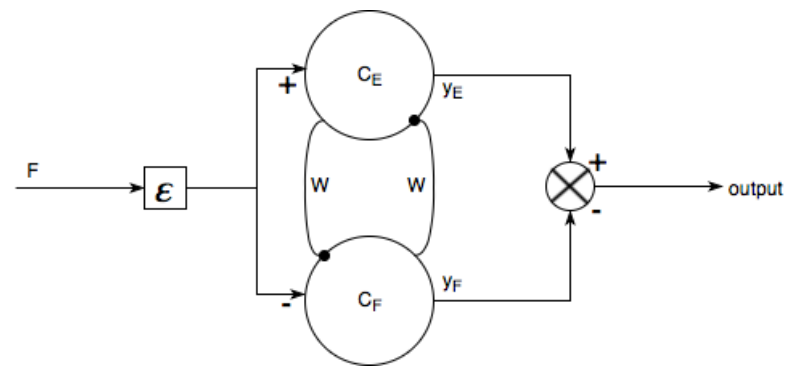

Fig. 1 Coupled oscillatory cells. $C_{\{E, F\}}$ indicates whether the neuron cell controls the extensor or the flexor

The equations are thus rewritten as follows (with $F_{F}=$ $\left.-F_{E}\right)$ :

\section{- Matsuoka model}

$$
\begin{aligned}
\dot{x}_{\{E, F\}}= & \frac{1}{T}\left(-x_{\{E, F\}}-W \cdot y_{\{F, E\}}-\right. \\
& \left.b \cdot v_{\{E, F\}}+c+\epsilon F_{\{E, F\}}\right) \\
\dot{v}_{\{E, F\}}= & \frac{1}{\tau}\left(-v_{\{E, F\}}+y_{\{E, F\}}\right) \\
y_{\{E, F\}}= & \max \left(x_{\{E, F\}}, 0\right)
\end{aligned}
$$

\section{- Hopf model}

$$
\begin{aligned}
\dot{x}_{\{E, F\}}= & \left(\mu-\left(x_{\{E, F\}}^{2}+y_{\{E, F\}}^{2}\right)\right) x_{\{E, F\}}- \\
& W \tanh \left(x_{\{F, E\}}\right)-\theta y_{\{E, F\}}+\epsilon F_{\{E, F\}} \\
\dot{y}_{\{E, F\}}= & \left(\mu-\left(x_{\{E, F\}}^{2}+y_{\{E, F\}}^{2}\right)\right) y_{\{E, F\}}+ \\
& \theta x_{\{E, F\}}
\end{aligned}
$$

Note that the term $W \tanh \left(x_{j}\right)$ has been added to the original model in order to couple the two Hopf cells and thus obtain a half center model. In the rest of the paper, we consider $W=1$ for Hopf.

\section{- Rowat-Selverston model}

$$
\begin{aligned}
\dot{V}_{\{E, F\}}= & y_{\{E, F\}}-W \frac{y_{\{E, F\}}}{1+e^{-4 y_{\{F, E\}}}} \\
& +\epsilon F_{\{E, F\}} \\
\dot{y}_{\{E, F\}}= & \frac{1}{\tau_{m}}\left(\sigma_{f}-\frac{\tau_{m}}{\tau_{s}}-1-\right. \\
& \left.\sigma_{f} \tanh ^{2}\left(\frac{\sigma_{f}}{A_{f}} V_{\{E, F\}}\right)\right) y_{\{E, F\}}- \\
& \frac{1+\sigma_{s}}{\tau_{s} \tau_{m}} V_{\{E, F\}}+ \\
& \frac{A_{f}}{\tau_{s} \tau_{m}} \tanh \left(\frac{\sigma_{f}}{A_{f}} V_{\{E, F\}}\right)
\end{aligned}
$$

\subsubsection{The Kasuga Control Architecture}

Kasuga and Hashimoto (2005) introduced a control architecture for human-robot handshaking using 
Matsuoka neurons. This framework was validated in simulation and experimentally. This architecture controls two robot joints which are coupled together (see Figure 2). The input of each neural oscillator is the weighted sum of the force exerted on the joint, absolute value of the force and output of the other joint controller. The force inputs determine the oscillation amplitude and whether the handshake is passive or active. Coupling the two joints together prevents the amplitude from decreasing, which has been observed with the Matsuoka oscillator by Kasuga and Hashimoto (2005). The output is considered as an angular velocity command.

In this system, the torque is applied by the ball to the gripper, which in turns entrains the robot joints and thus provides the CPG inputs. Because of the mechanical coupling, they form a closed loop with the human partner.

In order to integrate the oscillators into the Kasuga architecture for joints $i$ and $j$, the cells equations become:

\section{- Matsuoka model}

$$
\begin{aligned}
\dot{x}_{i_{\{E, F\}}=} & \frac{1}{T}\left(-x_{i_{\{E, F\}}}-W \cdot y_{i_{\{F, E\}}}-b \cdot v_{i_{\{E, F\}}}+c\right) \\
& +L_{i 2} F_{i_{\{E, F\}}}+L_{i 1}\left|F_{i_{\{E, F\}}}\right|+ \\
& K_{i_{\{E, F\}}}\left(y_{j_{E}}-y_{j_{F}}\right) \\
\dot{v}_{i_{\{E, F\}}=} & \frac{1}{\tau}\left(-v_{i_{\{E, F\}}}+y_{i_{\{E, F\}}}\right) \\
y_{i_{\{E, F\}}=} & \max \left(x_{\left.i_{\{E, F\}}, 0\right)}\right.
\end{aligned}
$$

With $F_{i_{E}}=-F_{i_{F}}, K_{i_{E}}=-K_{i_{F}}$ and $j$ the other joint.

\section{- Hopf model}

$$
\begin{aligned}
\dot{x}_{i_{\{E, F\}}=} & \left(\mu-\left(x_{i_{\{E, F\}}}^{2}+y_{i_{\{E, F\}}}^{2}\right)\right) x_{i_{\{E, F\}}} \\
& -\tanh \left(x_{i_{\{F, E\}}}\right)-\theta_{i} y_{i_{\{E, F\}}}+ \\
& L_{i 2} F_{i_{\{E, F\}}}+L_{i 1}\left|F_{i_{\{E, F\}}}\right|+ \\
& K_{i_{\{E, F\}}}\left(x_{j_{E}}-x_{j_{F}}\right) \\
\dot{y}_{i_{\{E, F\}}=} & \left(\mu-\left(x_{i_{\{E, F\}}}^{2}+y_{i_{\{E, F\}}}^{2}\right)\right) y_{i_{\{E, F\}}}+ \\
& \theta_{i} x_{i_{\{E, F\}}}
\end{aligned}
$$

\section{- Rowat-Selverston model}

$$
\begin{aligned}
& \dot{V}_{i_{\{E, F\}}}=y_{i_{\{E, F\}}}-W \frac{y_{i_{\{E, F\}}}}{1+e^{-4 y_{i_{\{F, E\}}}}}+ \\
& \cdot L_{i 2} F_{i_{\{E, F\}}}+L_{i 1}\left|F_{i_{\{E, F\}}}\right|+ \\
& K_{i_{\{E, F\}}}\left(V_{j_{E}}-V_{j_{F}}\right) \\
& \dot{y}_{i_{\{E, F\}}}=\frac{1}{\tau_{m}}\left(\sigma_{f}-\frac{\tau_{m}}{\tau_{s}}-1-\right. \\
& \left.\sigma_{f} \tanh ^{2}\left(\frac{\sigma_{f}}{A_{f_{i}}} V_{i_{\{E, F\}}}\right)\right) y_{i_{\{E, F\}}}- \\
& \frac{1+\sigma_{s}}{\tau_{s} \tau_{m}} V_{i_{\{E, F\}}}+ \\
& \frac{A_{f_{i_{\{E, F\}}}}}{\tau_{s} \tau_{m}} \tanh \left(\frac{\sigma_{f}}{A_{f_{i_{\{E, F\}}}}} V_{i_{\{E, F\}}}\right)
\end{aligned}
$$

Unless stated otherwise, the various gains are: $O_{i}=0.2$, $K_{i}=0.5, L_{i 2}=0.4, L_{i 1}=0.5$ for Rowat-Selverston and Hopf oscillators. For the Matsuoka oscillator, the input gains differ: $L_{i 2}=0.02, L_{i 1}=0.035$. Those values were obtained empirically. One important criterion was that the ball should stay inside the gripper. Indeed, some parameters created instability and unpredictable behavior from the robot. Then, the parameters were fine-tuned to try and get the best possible performance.

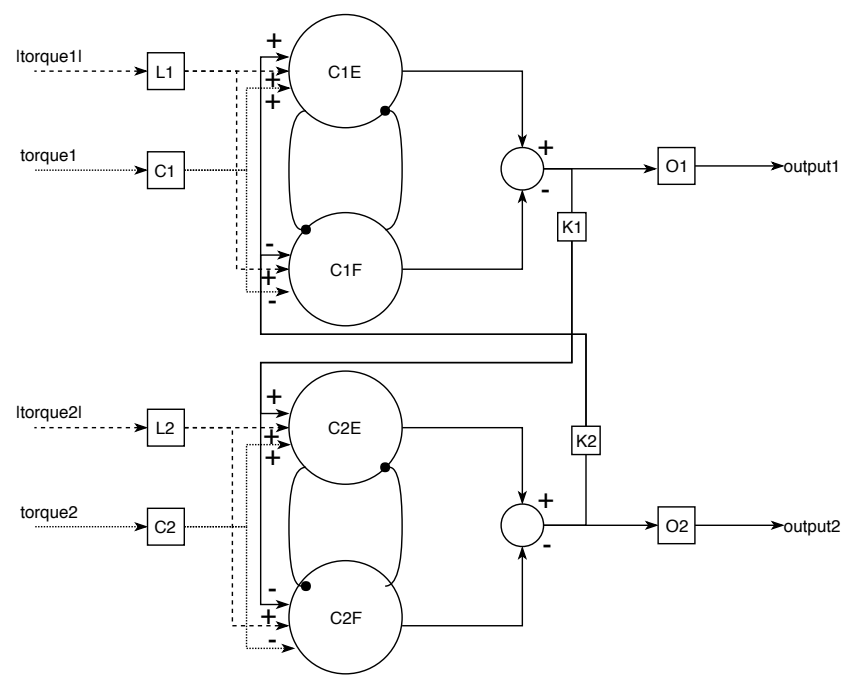

Fig. 2 Oscillatory cells integrated to the generalized Kasuga architecture. $C_{i}, L_{i}, K_{i}, O_{i}$ are gains and $C_{i\{E, F\}}$ the neuron cell for the joint $i$ controlling the extensor or flexor

\subsection{Evaluation of coordination: Phase Locking Value}

Introduced by Lachaux et al (1999) to measure coordination in brain signals, the Phase Locking Value (PLV) assumes that the two signals are locked with a constant phase difference. Allowing for deviations, it evaluates 
this spread: from 0 (no coordination) to 1 (perfect coordination). We use a variation of the original implementation. First, one has to obtain the instantaneous phase $\phi$ with the Hilbert transform, then the instantaneous PLV can be computed:

$P L V(t)=\frac{1}{N}\left|\sum_{i=0}^{N} e^{j\left(\phi_{1}(i)-\phi_{2}(i)\right)}\right|$

with $N$ the sliding window size, $j=\sqrt{-1}, \phi_{k}$ the instantaneous phase of signal $k$.

\section{Results: Comparison between three oscillator models}

The three oscillators are evaluated according to their entrainment range and their synchronization capacity during a handshaking simulation inspired by Kasuga and Hashimoto (2005). Then Hebbian plasticity is integrated to the Hopf and Rowat-Selverston models and the impact on the energetic cost is observed. We also implement a constants adaptation mechanism for Matsuoka.

\subsection{Entrainment Performance}

In order to determine their entrainment range, each oscillator model is subjected to various input frequencies for $100 \mathrm{~s}$. At the beginning, the input frequency is low at $0.1 \mathrm{~Hz}$ and increases by 0.1 every $100 \mathrm{~s}$. At each frequency change, the average PLV is computed between the input and output values of the oscillator, taking only the last $100 \mathrm{~s}$ into account. If this value is below 0.95 , the input frequency is considered outside the bandwidth of the oscillator.

The process is repeated for several intrinsic frequencies of the oscillators. Since the intrinsic frequency depends on the parameters, they are tuned in order to get matching frequencies: $\sigma_{s}, \theta$ and $b$ for Rowat-Selverston, Hopf and Matsuoka respectively.

Table 1 shows the entrainment range of each model for various intrinsic frequencies. We can clearly observe that the Matsuoka model is very limited and is not able to synchronize if the input frequency differs too much from its own. Besides, Rowat-Selverston cannot synchronize if the input frequency is too low, contrary to Hopf, but its frequency range is considerably larger.

Note that the entrainment capacity $\Delta \omega$ of Hopf is highly dependent on the value of $\epsilon$. The stronger the coupling, i.e the higher $\epsilon$, the better the oscillator synchronizes. On the contrary, $\epsilon$ has to be small for Matsuoka and neither too small, nor too big for RowatSelverston. Due to those discrepancies, we did not employ the same $\epsilon$ value for the oscillators but rather chose to put them all in the best possible synchronization conditions which were determined by running simulations with an extensive range of values of $\epsilon$, we then selected the value of $\epsilon$ which yielded the higher PLV score.

Table 1 The entrainment range where $P L V \geq 0.95$ for each oscillator with following parameters: Rowat-Selverston parameters: $\tau_{m}=0.35, \tau_{s}=3.5, \sigma_{f}=1.0, W=0.05, \epsilon=0.1$, $A_{f}=0.5$. Hopf parameters: $\mu=0.5, \epsilon=1.0$. Matsuoka parameters: $\tau=0.5, T=0.25, \epsilon=0.001, c=0.1, A=3 . \omega_{\text {intr }}$ is the intrinsic frequency of the neuron determined by $\sigma_{s}, \theta$ and $b$ respectively

\begin{tabular}{|c|c|c|c|c|c|}
\hline Model & & $\begin{array}{c}\omega_{\text {intr }} \\
{[\mathrm{Hz}]}\end{array}$ & $\begin{array}{c}\omega_{\min } \\
{[\mathrm{Hz}]}\end{array}$ & $\begin{array}{c}\omega_{\max } \\
{[\mathrm{Hz}]}\end{array}$ & $\begin{array}{c}\Delta \omega= \\
\omega_{\max }-\omega_{\min }\end{array}$ \\
\hline & $\sigma_{s}$ & & & & \\
\hline \multirow{6}{*}{$\begin{array}{l}\text { Rowat- } \\
\text { Selverston }\end{array}$} & 13 & 0.5 & 0.1 & 13.8 & 13.7 \\
\hline & 50 & 1.0 & 0.1 & 18.3 & 18.2 \\
\hline & 120 & 1.5 & 0.2 & 23.7 & 23.5 \\
\hline & 200 & 2.0 & 0.2 & 28.9 & 28.7 \\
\hline & 340 & 2.5 & 0.3 & 36.9 & 36.6 \\
\hline & 490 & 3.0 & 0.3 & 45.5 & 45.2 \\
\hline & $\theta$ & & & & \\
\hline \multirow{6}{*}{ Hopf } & 3.5 & 0.5 & 0.1 & 9.5 & 9.4 \\
\hline & 7 & 1.0 & 0.1 & 9.8 & 9.7 \\
\hline & 10 & 1.5 & 0.1 & 10.0 & 9.9 \\
\hline & 14 & 2.0 & 0.1 & 10.3 & 10.2 \\
\hline & 16 & 2.5 & 0.1 & 10.5 & 10.4 \\
\hline & 20 & 3.0 & 0.1 & 10.8 & 10.7 \\
\hline & $b$ & & & & \\
\hline \multirow{3}{*}{ Matsuoka } & 3.5 & 0.5 & 0.4 & 0.7 & 0.3 \\
\hline & 8 & 1.0 & 0.9 & 1.2 & 0.3 \\
\hline & 13 & 1.5 & 1.3 & 1.6 & 0.3 \\
\hline
\end{tabular}

\subsection{Synchronization Evaluation in Simulated} Handshaking

In this section, in order to evaluate the three oscillators, they are placed into the version of the Kasuga architecture introduced previously in section 2.2 .2 , which has been generalized such that any oscillator can be integrated.

The simulations have been conducted with the Kinova Mico robot in the V-REP simulator. Since grasping cannot accurately be simulated, we realize a oversimplified handshake with a static collidable ball instead of the human hand. The ball is animated with an up and down motion of amplitude $0.16 \mathrm{~m}$ and frequency $2 \mathrm{~Hz}$, unless stated otherwise. The $2 \mathrm{~Hz}$ frequency was found appropriate for handshaking (Tagne et al, 2016). The moving ball forces the arm to move along, by ex- 
erting a force on the fingers of the robot (see Figure $3)$.

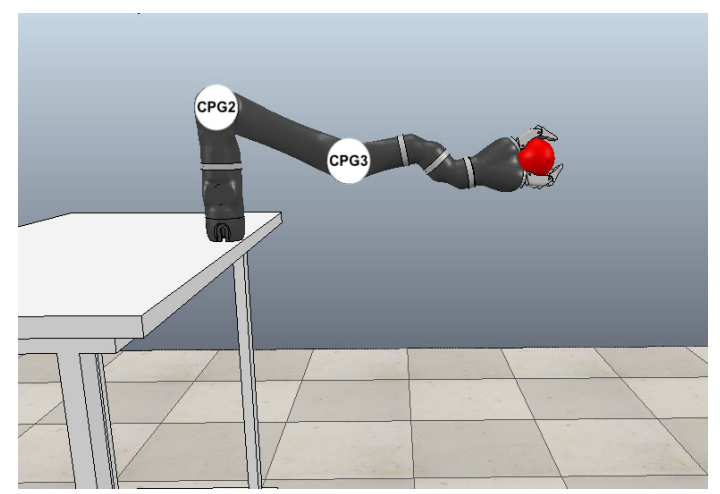

Fig. 3 Simulated robotic arm with the static ball inside its gripper. We control the ball kinematics. We consider a rigid mechanical link because we want to impose a force on the robot which cannot be influenced. We set the ball position repeatedly according to a sinusoidal signal. The time step is small enough so that the motion appears smooth

The Mico arm has six degrees of freedom, but in the current setup, inspired by Kasuga and Hashimoto (2005), two joints, only the shoulder and elbow (joints 2 and 3 of the Mico robot) are controlled, the four other joints are locked. At the beginning of the simulation, the robot is not subjected to any external force (other than gravity). Then, the ball moves in the vertical plane, applying a perturbation to the robotic arm. Finally, the interaction stops and the ball is released.

In order to evaluate how the oscillators react when subjected to various frequency changes, the input signal applied to the ball varies across time: from $t=0 \mathrm{~s}$ to $\mathrm{t}$ $=20 \mathrm{~s}, 0.5 \mathrm{~Hz}$; from $\mathrm{t}=20 \mathrm{~s}$ to $\mathrm{t}=40 \mathrm{~s}, 1.0 \mathrm{~Hz}$; from $\mathrm{t}=40 \mathrm{~s}$ to $\mathrm{t}=60 \mathrm{~s}, 1.5 \mathrm{~Hz}$; from $\mathrm{t}=60 \mathrm{~s}$ to $\mathrm{t}=80$ $\mathrm{s}, 2.0 \mathrm{~Hz}$, from $\mathrm{t}=80 \mathrm{~s}$ to $\mathrm{t}=100 \mathrm{~s}, 1.5 \mathrm{~Hz}$; at $\mathrm{t}=$ $100 \mathrm{~s}$, the ball is released and until $\mathrm{t}=110 \mathrm{~s}$, no force is applied.

\subsubsection{Results without any Plasticity Mechanisms}

At the beginning of the interaction, the intrinsic frequency of each oscillator is $1 \mathrm{~Hz}$. See Table 2 for the parameters used in the simulations.

Table 2 Oscillator parameters.

\begin{tabular}{|c||c|}
\hline Model & Parameters \\
\hline Matsuoka & $\tau=0.5, T=0.25, c=0.1$, \\
& $W=4.0, b=10.0$ \\
\hline Rowat- & $\tau_{m}=0.2, \tau_{s}=2.0, \sigma_{f}=1.0, W=0.05$, \\
Selverston & $A_{f_{2}}=0.5, A_{f_{3}}=0.9, \sigma_{s}=20$ \\
\hline Hopf & $\mu=0.5, \theta=7.0$ \\
\hline
\end{tabular}

Figures 4, 5 and 6 represent the $\mathrm{CPG}$ input and output over $3 \mathrm{~s}$ for each frequency range. Matsuoka appears unable to synchronize at $0.5 \mathrm{~Hz}$ and the motion is irregular during the whole simulation (Figure 4). Kasuga and Hashimoto (2005)'s results had a smooth and regular motion because the oscillator was put in a non-oscillating mode where the Matsuoka oscillator synchronizes better. Hopf and Rowat-Selverston, however, have satisfactory frequency entrainment and the motion is regular, except for $0.5 \mathrm{~Hz}$ where Hopf struggles to synchronize (Figure 5). For the three oscillators, we can also observe that the input signal slightly precedes the output signal.

The PLV, represented on Figure 7 is used to evaluate the coordination between the force exerted on the joints and each neural oscillator model. This confirms what we previously observed: while the Matsuoka PLV (mean PLV: 0.91, 0.86) appears satisfactory, it actually never reaches 1.0 and is highly irregular for $0.5 \mathrm{~Hz}$. This can be explained by the fact that the PLV quality and accuracy decrease when the signals are not perfectly sinusoidal. The PLV of Rowat-Selverston (0.93, $0.93)$ is more regular than Hopf $(0.91,0.9)$ for $0.5 \mathrm{~Hz}$, their performances are similar for the rest of the simulation, though the transitions are more noticeable for Rowat-Selverston.

\subsubsection{Results with Plasticity Mechanisms}

In this section, Hebbian plasticity mechanisms are integrated to Hopf and Rowat-Selverston in order to have more adaptable and versatile systems. For Matsuoka, a time constants adaptation mechanism is implemented. Then, the oscillators are once more evaluated.

Simulations were run with the same parameters as previously. Additionally, for Rowat-Selverston, $\lambda=0.005, \mu=5 e^{-6} ; \epsilon_{2}=0.2, \epsilon_{3}=0.3$ and for Hopf, $\eta_{2}=0.2, \eta_{3}=0.25$. Besides, plasticity could not be applied with the previous Kasuga parameters, $L_{i 1}$ had to be set to zero. For Matsuoka, $C_{i}=0.08$ and $K_{i}=0.1$.

Figures 8, 9, 10 and 11 represent the CPG input and output over $3 \mathrm{~s}$ for each frequency range. Plasticity has little effect on the PLV (see Figure 12). The Matsuoka PLV (mean PLV: 0.86, 0.84) still never reaches 1.0 and is highly irregular for $0.5 \mathrm{~Hz}$ and $1 \mathrm{~Hz}$. RowatSelverston with frequency learning $(0.93,0.92)$ and all plasticities $(0.92,0.89)$ is similar to Hopf $(0.90,0.89)$ overall. The PLV appears to decrease for joint 3 with Rowat-Selverston with plasticities, however, this can be explained by the fact that the force decreases so much, it becomes mostly noise, which the PLV does not handle well. 


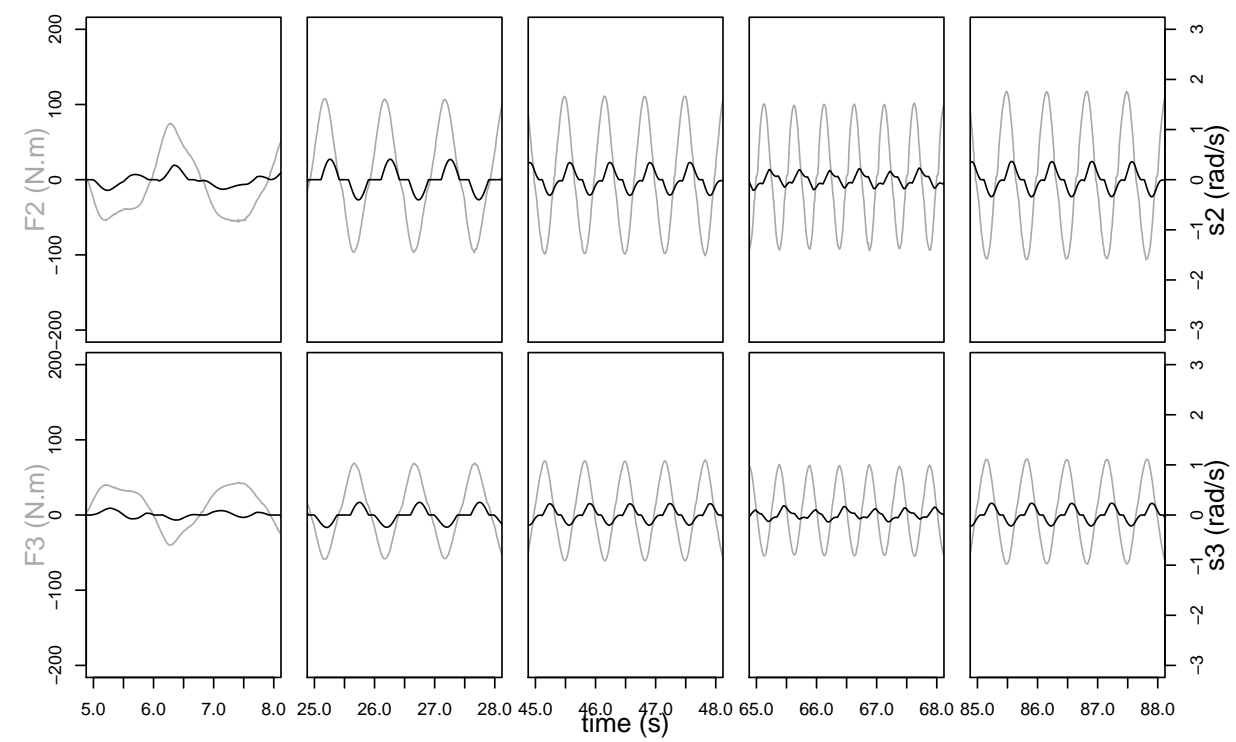

Figure 4 Response of the Matsuoka oscillator. In gray: CPG input, in black: CPG output for both joints

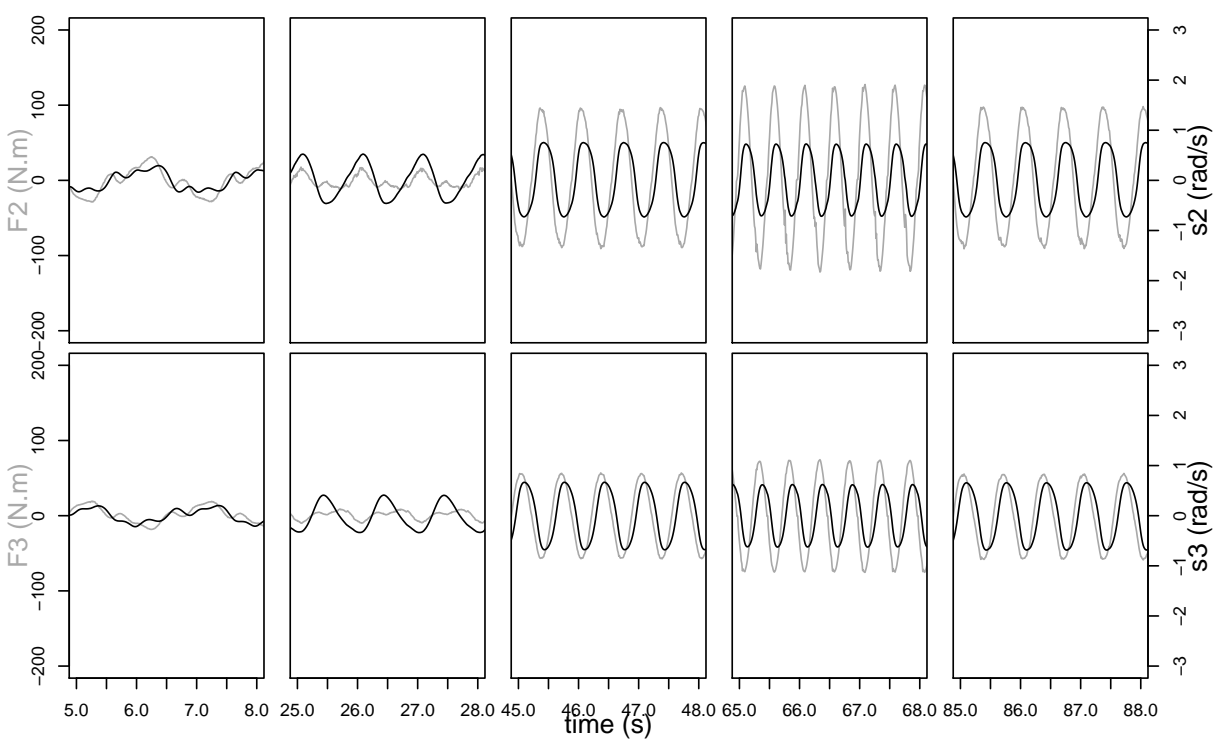

Figure 5 Response of the Hopf oscillator. In gray: CPG input, in black: CPG output for both joints

However, plasticity slightly extends the entrainment range (see Table 3), especially for Matsuoka which now has an entrainment range similar to Hopf. Furthermore it particularly improves the energetic impact of the oscillator. Indeed, if the intrinsic properties (amplitude, intrinsic frequency) of the oscillator are able to adapt to a varying input by learning more suitable parameters, the ball will be able to anticipate the input signal and will not exert so much force on the robotic arm and hence the amplitude of the force applied would be less important. This anticipation is evidenced by the fact that the delay between the input and output has now disappeared for Hopf and Rowat-Selverston. It is however still present for Matsuoka. Table 4 shows that frequency learning already leads to a significant decrease of the average power consumed by the robot for all three oscillators. Furthermore, $A_{f}$ and $\epsilon$ learning for Rowat-Selverston decrease this consumption further.

Comparing with the simulations without plasticity, we see that the force applied on the joints for the three oscillators without plasticity never decreased. This shows that while the oscillator seems to adapt, it merely moves along, entrained by the ball, hence the delay between the input and output. This is further illustrated by the fact that once the interaction is over, the oscillator does not retain the ball frequency but returns to its own. On the other hand, for the oscillators endowed with plasticity, once they adapt 


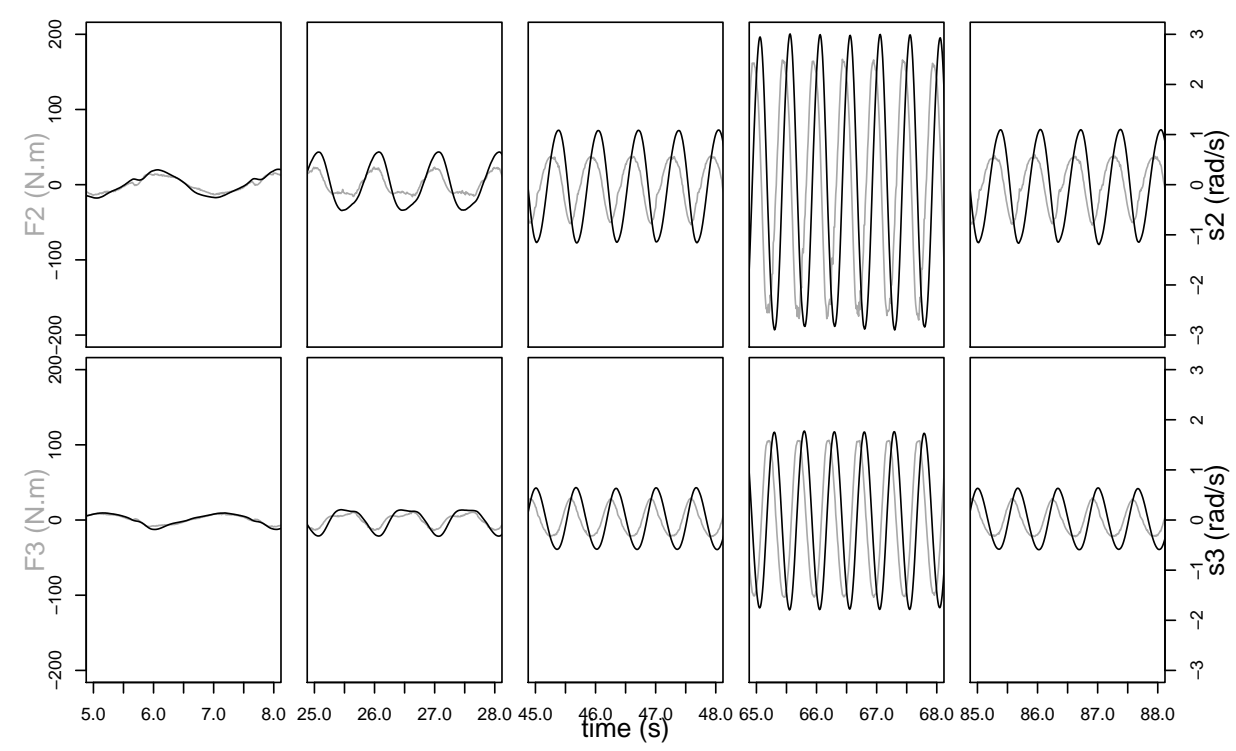

Figure 6 Response of the Rowat-Selverston oscillator. In gray: CPG input, in black: CPG output for both joints
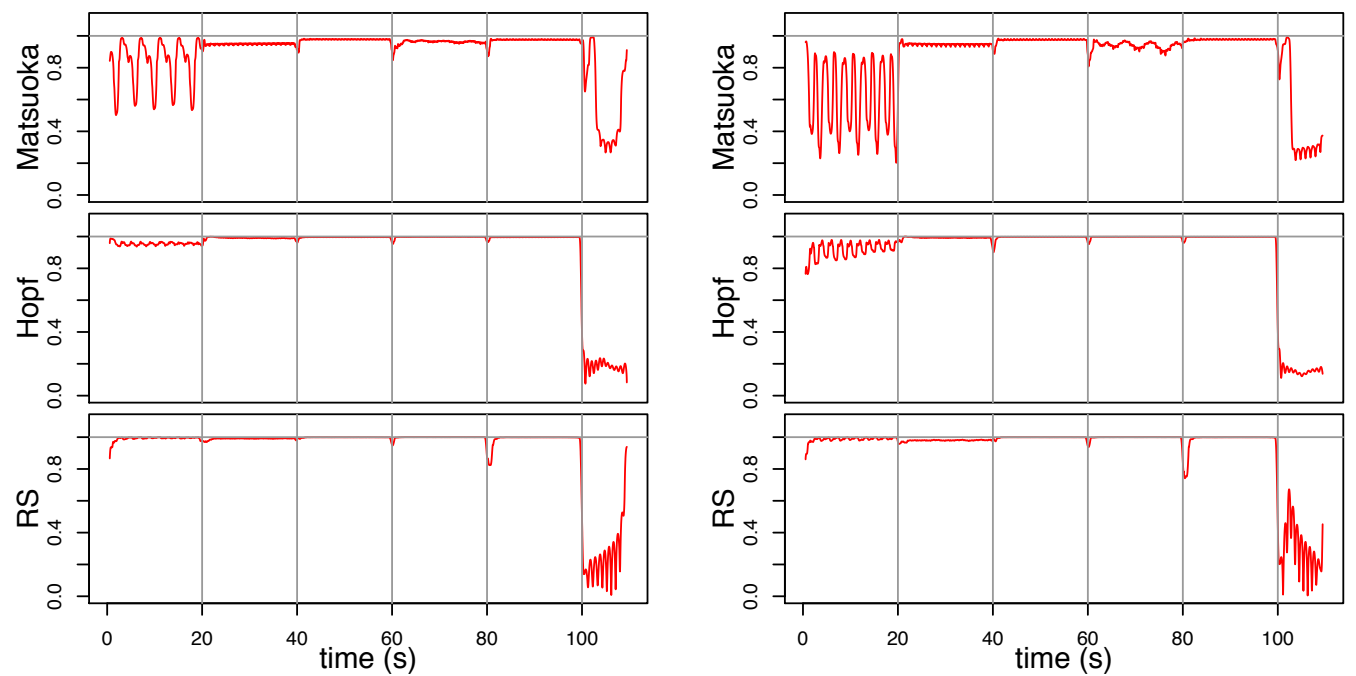

Figure 7 PLV for joint 2 (left), joint 3 (right). The vertical grey lines delimit each frequency range. The horizontal line indicates the reference for perfect coordination at 1.0

(new frequency learned), the delay disappears and a decrease in force can be observed, even more so for the Rowat-Selverston oscillator with all plasticities.

\section{Discussion}

In this paper, we compared three oscillators often used in CPG modeling: Matsuoka, Hopf and RowatSelverston. These oscillators were evaluated firstly on their entrainment range. Then they were integrated into a simple control architecture and we compared their synchronization and power consumption performance with and without plasticity using a handshaking simulation.
We showed that integrating plasticity mechanisms into the three oscillators has no real impact on coordination performance but can lead to a significant power consumption decrease and an extended entrainment range.

With a cumbersome parameter tuning process, a very limited entrainment range, the Matsuoka oscillator is not the best choice for the adaptive control of a robot confronted to unpredictable feedbacks. Indeed, one has to bear in mind that CPG use is not restricted to human handshaking. To get good performance with the Matsuoka oscillator one has to have a rather accurate idea of the input (frequency, amplitude) beforehand. However, the time constants adaptation mechanism significantly improves the entrainment of this oscillator and even the power consumption. 


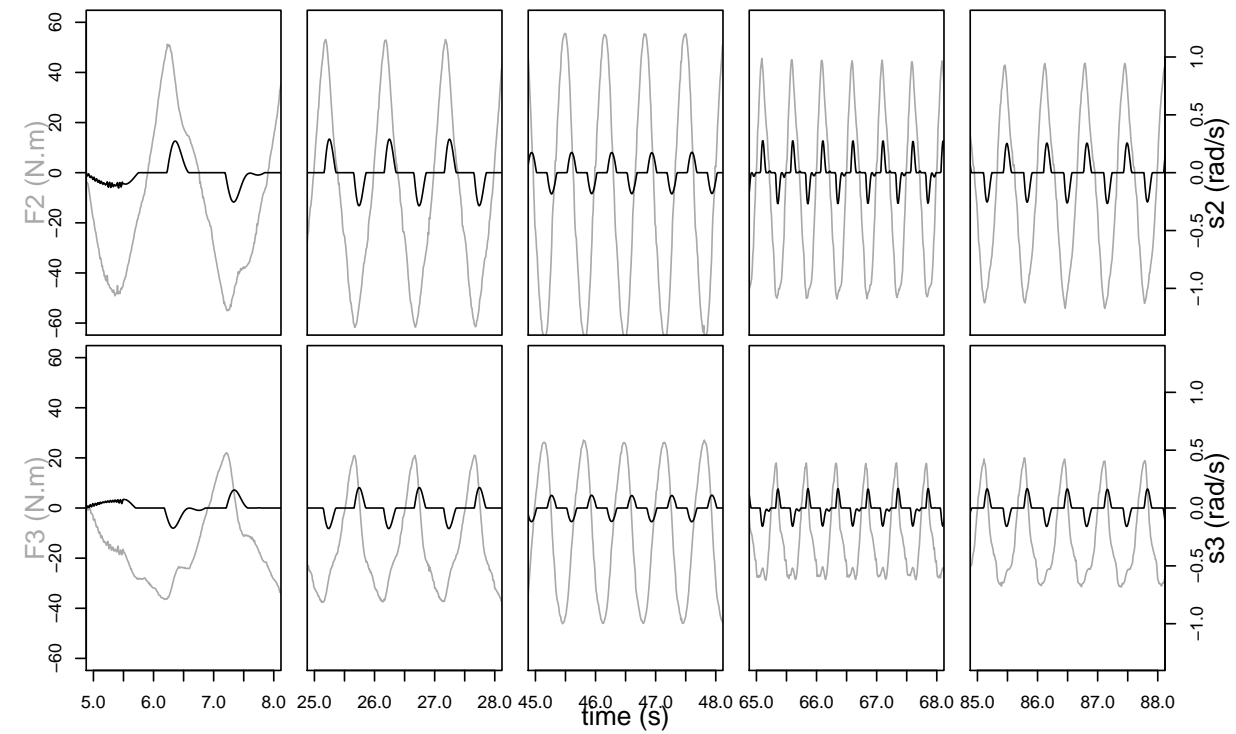

Figure 8 Response of the Matsuoka oscillator with time constants adaptation. In gray: CPG input, in black: CPG output for both joints

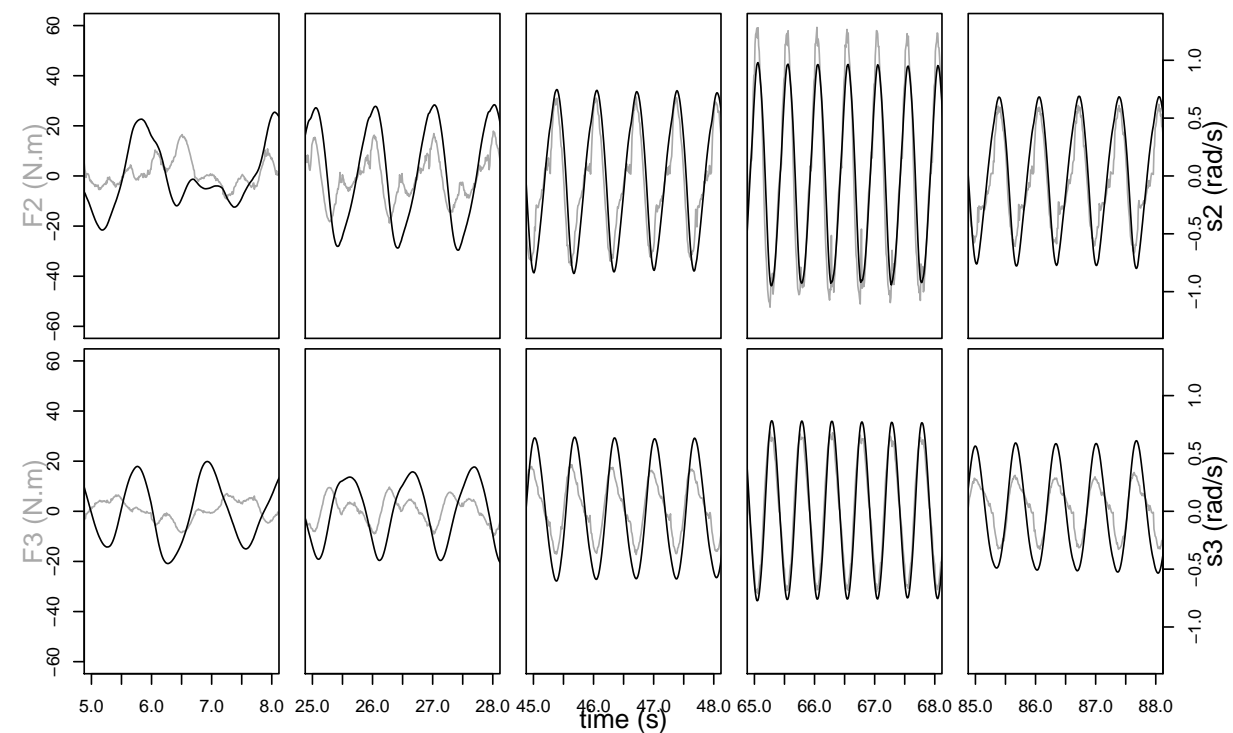

Figure 9 Response of the Hopf oscillator with Frequency Learning. In gray: CPG input, in black: CPG output for both joints

Comparing Hopf and Rowat-Selverston is however less straightforward. While Hopf synchronizes as well (sometimes better) as Rowat-Selverston and appears easier to control, this simplicity makes it less than obvious to render Hopf more adaptive by integrating new plasticity rules. Its entrainment range is noticeably smaller, even with frequency learning and its power consumption greater than Rowat-Selverston. It could probably benefit from amplitude learning however, amplitude setting is done in such a way that we fail to see how it could possibly be learned.

On the other hand, Rowat-Selverston is obviously more complex with more parameters to handle but it offers more flexibility and versatility, making it possible to design plasticity mechanisms allowing the system to truly adapt and not only offer a wide entrainment range but also a significantly lower power consumption.

Furthermore, the three oscillators are all non-linear systems, however the degree of non-linearity can be modified more easily for Rowat-Selverston and Hopf than Matsuoka. Rowat-Selverston also has a discrete mode where it behaves like a PID controller (Jouaiti and Henaff, 2018). This, however, does not seem to be possible for Hopf and Matsuoka.

We feel that this study can help understand these oscillators better and for which purpose they are better 


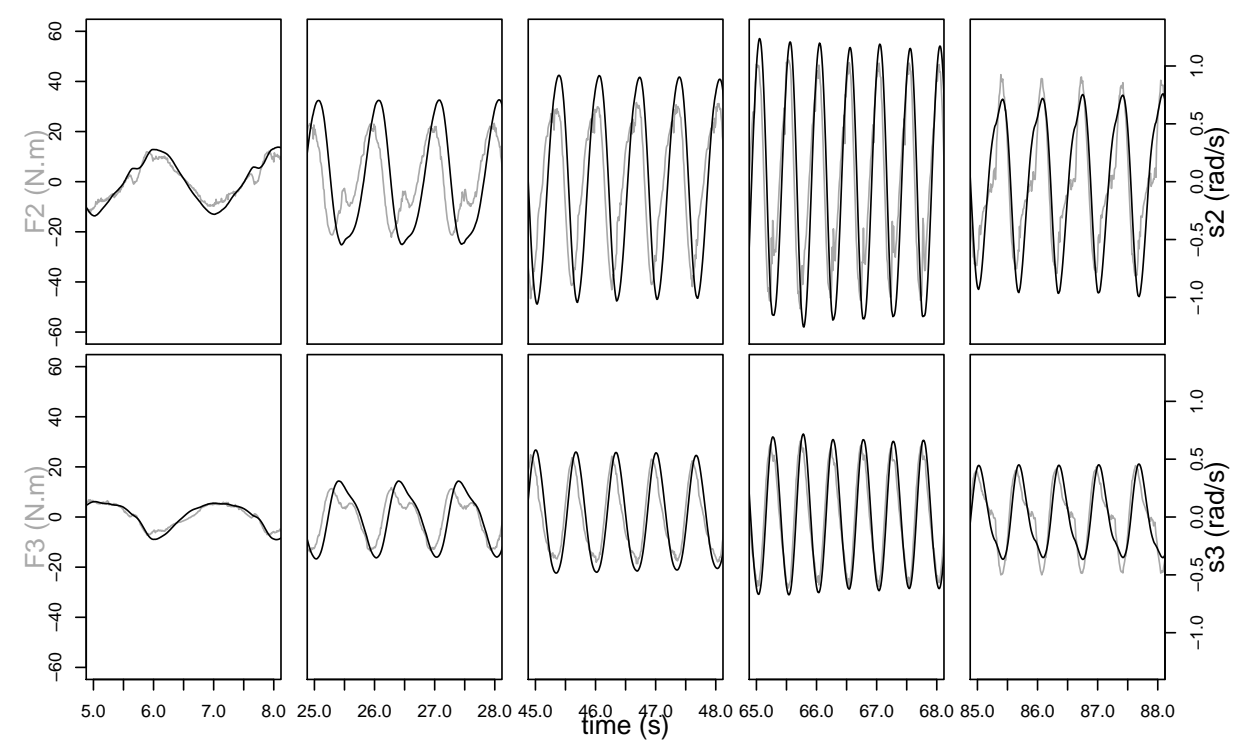

Figure 10 Response of the Rowat-Selverston oscillator with Frequency Learning. In gray: CPG input, in black: CPG output for both joints

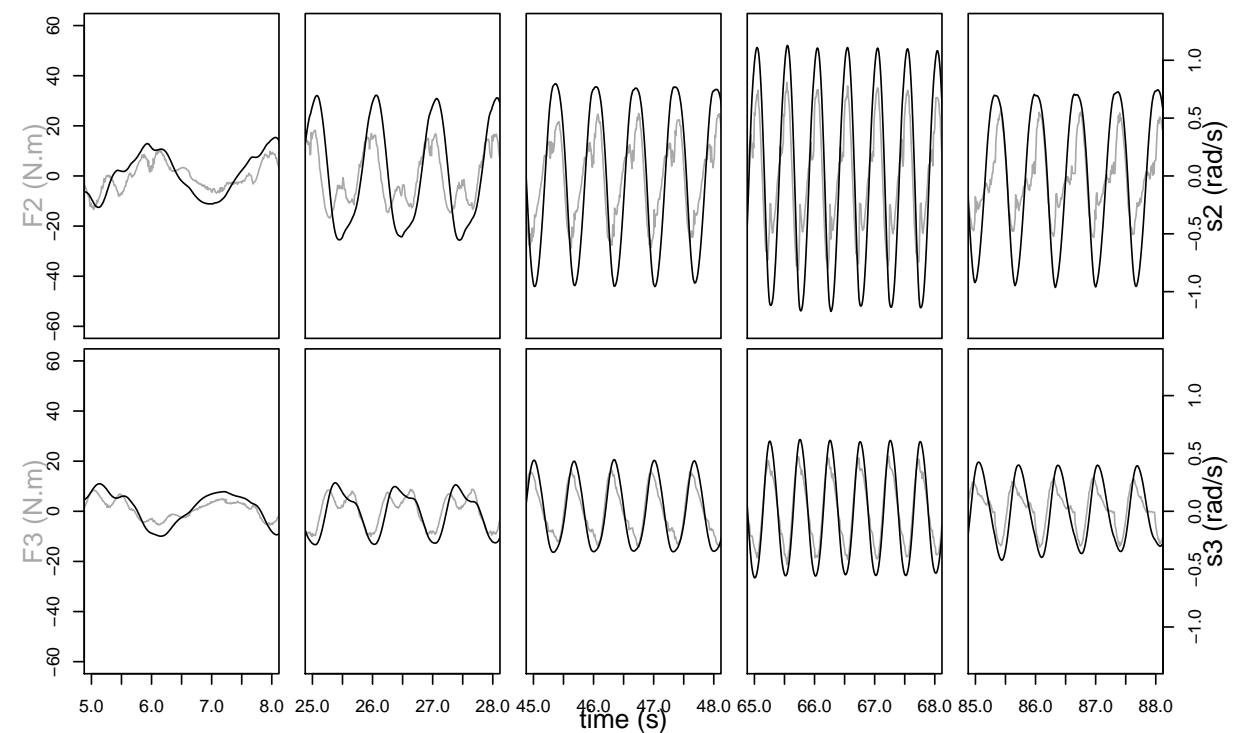

Figure 11 Response of the Rowat-Selverston oscillator with all the plasticity mechanisms. In gray: CPG input, in black: CPG output for both joints

suited. Very few works undertook the oscillator comparison endeavour (Collins and Richmond, 1994). We, however, think that this is an important question as many models are available but each has its own strength and weaknesses and they may be suitable for different purposes. In this work, we arbitrarily chose the Matsuoka, Hopf and Rowat-Selverston models because we see them as complementary. Nevertheless, this work could be extended by integrating other models into the comparison, and also by testing other applications such as locomotion, rhythmic work tasks, rhythmic movements with no physical interaction and so on and so forth. In future works, it would also be interesting to evaluate the impact of impedance control on the oscillators and particularly the feel of the interaction in human-robot physical interactions. We also expect the stiffness to influence the learning mechanisms, especially the adaptation speed. 

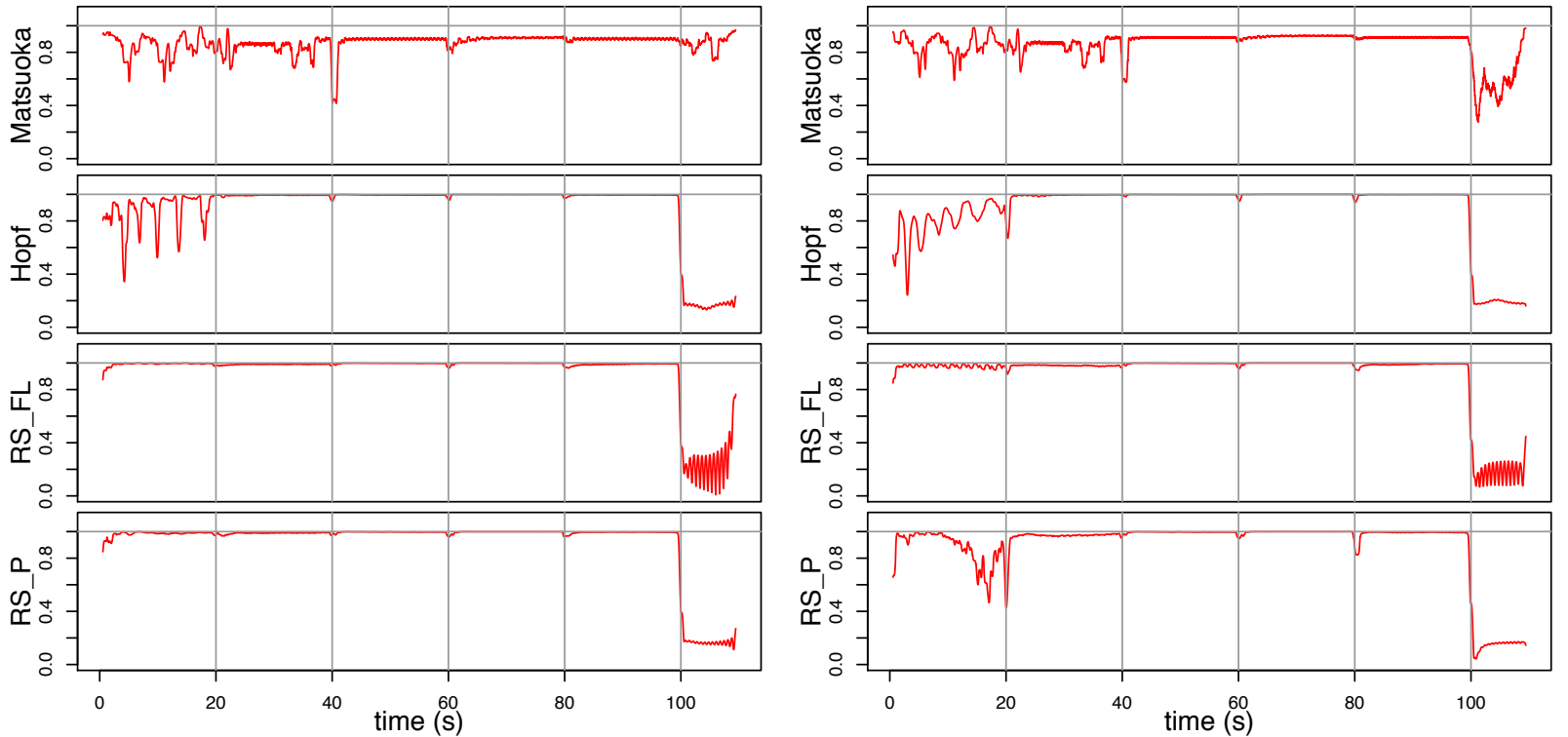

Figure 12 PLV for joint 2 (left), joint 3 (right). The vertical grey lines delimit each frequency range. The horizontal line indicates the reference for perfect coordination at 1.0

Table 3 Synchrony frequency range where $P L V \geq 0.95$ for each oscillator with plasticity. $\Delta \omega_{1}$ and $\Delta \omega_{2}$ define the frequency entrainment with plasticity and without plasticity

\begin{tabular}{|c|c|c|c|c|c|c|}
\hline Model & & $\begin{array}{c}\omega_{\text {intr }} \\
{[\mathrm{Hz}]}\end{array}$ & $\begin{array}{c}\omega_{\min } \\
{[\mathrm{Hz}]}\end{array}$ & $\begin{array}{c}\omega_{\max } \\
{[\mathrm{Hz}]}\end{array}$ & $\Delta \omega_{1}$ & $\Delta \omega_{2}$ \\
\hline & $\sigma_{s}$ & & & & & \\
\hline \multirow{6}{*}{$\begin{array}{l}\text { Rowat- } \\
\text { Selverston } \\
\text { Frequency } \\
\text { Learning }\end{array}$} & 13 & 0.5 & 0.1 & 14.1 & 14 & 13.7 \\
\hline & 50 & 1.0 & 0.1 & 18.6 & 18.5 & 18.2 \\
\hline & 120 & 1.5 & 0.1 & 24.1 & 24 & 23.5 \\
\hline & 200 & 2.0 & 0.1 & 29.2 & 29.1 & 28.7 \\
\hline & 340 & 2.5 & 0.1 & 37.5 & 37.4 & 36.6 \\
\hline & 490 & 3.0 & 0.1 & 46.6 & 46.5 & 45.2 \\
\hline & $\theta$ & & & & & \\
\hline \multirow{6}{*}{$\begin{array}{c}\text { Hopf } \\
\text { Frequency } \\
\text { Learning }\end{array}$} & 3.5 & 0.5 & 0.1 & 13.6 & 13.5 & 9.4 \\
\hline & 7 & 1.0 & 0.1 & 14.0 & 13.9 & 9.7 \\
\hline & 10 & 1.5 & 0.1 & 14.2 & 14.1 & 9.9 \\
\hline & 14 & 2.0 & 0.1 & 14.7 & 14.6 & 10.2 \\
\hline & 16 & 2.5 & 0.1 & 14.9 & 14.8 & 10.4 \\
\hline & 20 & 3.0 & 0.1 & 15.5 & 15.4 & 10.7 \\
\hline & b & & & & & \\
\hline \multirow{3}{*}{$\begin{array}{c}\text { Matsuoka } \\
T, \tau \\
\text { Adaptation } \\
\end{array}$} & 3.5 & 0.5 & 0.1 & 10.1 & 10.0 & 0.3 \\
\hline & 8 & 1.0 & 0.2 & 14.9 & 13.7 & 0.3 \\
\hline & 13 & 1.5 & 0.3 & 13.6 & 13.3 & 0.3 \\
\hline
\end{tabular}

Table 4 Average power $\frac{1}{T_{\max }} \sum\left|F_{i}(t) \cdot \operatorname{vel}(t)\right|$ (Watt) applied to both joints using the various neural oscillators

\begin{tabular}{|c|c|c|}
\hline Model & Joint 2 & Joint 3 \\
\hline Rowat-Selverston & 2.15 & 1.41 \\
Rowat-Selverston $-\sigma_{s}$ Learning & 0.33 & 0.20 \\
Rowat-Selverston $-\sigma_{s}, A_{f}, \epsilon$ Learning & 0.23 & 0.10 \\
\hline Hopf & 1.12 & 1.07 \\
Hopf $-\theta$ Learning & 0.34 & 0.24 \\
\hline Matsuoka & 1.16 & 0.27 \\
Matsuoka - Time constants adaptation & 0.54 & 0.1 \\
\hline
\end{tabular}

\section{References}

Al-Busaidi AM, Zaier R, Al-Yahmadi AS (2012) Control of biped robot joints' angles using coordinated matsuoka oscillators. In: International Conference on Artificial Neural Networks, Springer, pp 304-312

Arena P, Fortuna L, Frasca M, Patane L, Pollino M (2006) An autonomous mini-hexapod robot controlled through a cnn-based cpg vlsi chip. In: Cellular Neural Networks and Their Applications, 2006. CNNA'06. 10th International Workshop on, IEEE, pp $1-6$

Arikan KB, Irfanoglu B (2011) A test bench to study bioinspired control for robot walking. Journal of Control Engineering and Applied Informatics 13(2):76-80

Ayers J (2004) Underwater walking. Arthropod structure \& development 33(3):347-360

Brambilla G, Buchli J, Ijspeert AJ (2006) Adaptive four legged locomotion control based on nonlinear dynamical systems. In: International Conference on Simulation of Adaptive Behavior, Springer, pp 138-149

Buchli J, Ijspeert AJ (2008) Self-organized adaptive legged locomotion in a compliant quadruped robot. Autonomous Robots 25(4):331

Buchli J, Righetti L, Ijspeert AJ (2005) A dynamical systems approach to learning: a frequency-adaptive hopper robot. In: European Conference on Artificial Life, Springer, pp 210-220

Buchli J, Iida F, Ijspeert AJ (2006) Finding resonance: Adaptive frequency oscillators for dynamic legged locomotion. In: Intelligent robots and systems, 2006 ieee/rsj international conference on, IEEE, pp 39033909

Cattaert D, Le Ray D (2001) Adaptive motor control in crayfish. Progress in neurobiology 63(2):199-240

Chung SJ, Dorothy M (2010) Neurobiologically inspired control of engineered flapping flight. Journal of guid- 
ance, control, and dynamics 33(2):440-453

Collins JJ, Richmond SA (1994) Hard-wired central pattern generators for quadrupedal locomotion. Biological Cybernetics 71(5):375-385

Degallier S, Santos CP, Righetti L, Ijspeert A (2006) Movement generation using dynamical systems: a humanoid robot performing a drumming task. In: Humanoid Robots, 2006 6th IEEE-RAS International Conference on, IEEE, pp 512-517

Degallier S, Righetti L, Natale L, Nori F, Metta G, Ijspeert A (2008) A modular bio-inspired architecture for movement generation for the infant-like robot icub. In: Biomedical Robotics and Biomechatronics, 2008. BioRob 2008. 2nd IEEE RAS \&amp; EMBS International Conference on, IEEE, pp 795-800

Fang F, Xu WL, Lin K, Alam F, Potgieter J (2013) Matsuoka neuronal oscillator for traffic signal control using agent-based simulation. Procedia Computer Science 19:389-395

Fuente LA, Lones MA, Turner AP, Caves LS, Stepney S, Tyrrell AM (2013) Adaptive robotic gait control using coupled artificial signalling networks, hopf oscillators and inverse kinematics. In: Evolutionary Computation (CEC), 2013 IEEE Congress on, IEEE, pp 1435-1442

Grillner S, Wallen P (1985) Central pattern generators for locomotion, with special reference to vertebrates. Annual review of neuroscience 8(1):233-261

He J, Lu C, Yin S (2006) The design of cpg control module of the bionic mechanical crab. In: Robotics and Biomimetics, 2006. ROBIO'06. IEEE International Conference on, IEEE, pp 280-285

Hodgkin AL, Huxley AF (1952) A quantitative description of membrane current and its application to conduction and excitation in nerve. The Journal of physiology 117(4):500-544

Hopf E (1942) Abzweigung einer periodischen lösung von einer stationären lösung eines differentialsystems. Ber Math-Phys Kl Sächs Akad Wiss Leipzig 94:1-22

$\mathrm{Hu}$ Y, Tian W, Liang J, Wang T (2011) Learning fish-like swimming with a cpg-based locomotion controller. In: Intelligent Robots and Systems (IROS), 2011 IEEE/RSJ International Conference on, IEEE, pp 1863-1868

$\mathrm{Hu}$ Y, Liang J, Wang T (2014) Parameter synthesis of coupled nonlinear oscillators for cpg-based robotic locomotion. IEEE Transactions on Industrial Electronics 61(11):6183-6191

Ijspeert AJ (2008) Central pattern generators for locomotion control in animals and robots: A review. Neural networks 21(4):642-653
Ijspeert J (2004) A simple adaptive locomotion toysystem. In: From Animals to Animats 8: Proceedings of the Seventh [ie Eighth] International Conference on Simulation of Adaptive Behavior, MIT Press, vol 8, p 153

Jouaiti M, Henaff P (2018) Cpg-based controllers can generate both discrete and rhythmic movements. In: 2018 IEEE/RSJ International Conference on Intelligent Robots and Systems (IROS)

Jouaiti M, Caron L, Hénaff P (2018) Hebbian plasticity in cpg controllers facilitates self-synchronization for human-robot handshaking. Frontiers in Neurorobotics 12:29

Kamimura A, Kurokawa H, Yoshida E, Murata S, Tomita K, Kokaji S (2005) Automatic locomotion design and experiments for a modular robotic system. IEEE/ASME Transactions on mechatronics 10(3):314-325

Kasuga T, Hashimoto M (2005) Human-robot handshaking using neural oscillators. In: Robotics and Automation, 2005. ICRA 2005. Proceedings of the 2005 IEEE International Conference on, IEEE, pp 3802 3807

Lachaux JP, Rodriguez E, Martinerie J, Varela FJ, et al (1999) Measuring phase synchrony in brain signals. Human brain mapping 8(4):194-208

Li C, Lowe R, Ziemke T (2013) Humanoids learning to walk: a natural cpg-actor-critic architecture. Frontiers in neurorobotics 7:5

Liu C, Chen Q, Wang D (2011) Cpg-inspired workspace trajectory generation and adaptive locomotion control for quadruped robots. IEEE Transactions on Systems, Man, and Cybernetics, Part B (Cybernetics) 41(3):867-880

Liu C, Fan Z, Seo K, Tan X, Goodman E (2012) Synthesis of matsuoka-based neuron oscillator models in locomotion control of robots. In: Intelligent Systems (GCIS), 2012 Third Global Congress on, IEEE, pp 342-347

Liu GL, Watanabe K, Izumi K (2006) The parameter design of central pattern generators composed of some matsuoka oscillators for the leg movements of human-like robots. In: SCIS \&amp; ISIS SCIS \&amp; ISIS 2006, Japan Society for Fuzzy Theory and Intelligent Informatics, pp 24-29

Liu GL, Habib MK, Watanabe K, Izumi K (2007) The design of central pattern generators based on the matsuoka oscillator to generate rhythmic humanlike movement for biped robots. Journal of Advanced Computational Intelligence and Intelligent Informatics $11(8)$

Liu GL, Habib MK, Watanabe K, Izumi K (2008) Central pattern generators based on matsuoka oscillators 
for the locomotion of biped robots. Artificial Life and Robotics 12(1-2):264-269

Manoonpong P, Pasemann F, Wörgötter F (2008) Sensor-driven neural control for omnidirectional locomotion and versatile reactive behaviors of walking machines. Robotics and Autonomous Systems 56(3):265-288

Matos V, Santos C (2010) Omnidirectional locomotion in a quadruped robot: A cpg-based approach. In: The 2010 IEEE/RSJ International Conference on Intelligent Robots and Systems, IROS 2010, pp 3392-3397

Matsuoka K (1985) Sustained oscillations generated by mutually inhibiting neurons with adaptation. Biological cybernetics 52(6):367-376

Matsuoka K (2011) Analysis of a neural oscillator. Biological cybernetics 104(4):297-304

Mori T, Nakamura Y, Sato MA, Ishii S (2004) Reinforcement learning for cpg-driven biped robot. In: AAAI, vol 4, pp 623-630

Nassour J, Hénaff P, Benouezdou F, Cheng G (2014) Multi-layered multi-pattern cpg for adaptive locomotion of humanoid robots, biological cybernetics. Biological cybernetics 108(3):291-303

Nassour J, Hoa TD, Atoofi P, Hamker F (2019) Concrete action representation model: from neuroscience to robotics. IEEE Transactions on Cognitive and Developmental Systems

Panwart V, Kumar R (2012) Stable biped locomotion using central pattern generators based on matsuoka neural oscillators

Pelc EH, Daley MA, Ferris DP (2008) Resonant hopping of a robot controlled by an artificial neural oscillator. Bioinspiration \& biomimetics 3(2):026001

Pinto CM, Rocha D, Santos CP (2012) Hexapod robots: new cpg model for generation of trajectories. J Numer Anal Ind Appl Math 7(1-2):15-26

Righetti L, Ijspeert AJ (2006) Programmable central pattern generators: an application to biped locomotion control. In: Robotics and Automation, 2006. ICRA 2006. Proceedings 2006 IEEE International Conference on, IEEE, pp 1585-1590

Righetti L, Ijspeert AJ (2008) Pattern generators with sensory feedback for the control of quadruped locomotion. In: Robotics and Automation, 2008. ICRA 2008. IEEE International Conference on, IEEE, pp 819-824

Righetti L, Buchli J, Ijspeert AJ (2006) Dynamic hebbian learning in adaptive frequency oscillators. Physica D: Nonlinear Phenomena 216(2):269-281

Rowat PF, Selverston AI (1993) Modeling the gastric mill central pattern generator of the lobster with a relaxation-oscillator network. Journal of neurophysiology 70(3):1030-1053 de Rugy A, Wei K, Müller H, Sternad D (2003) Actively tracking passive stability in a ball bouncing task. Brain research 982(1):64-78

Rybak IA, Shevtsova NA, Lafreniere-Roula M, McCrea DA (2006) Modelling spinal circuitry involved in locomotor pattern generation: insights from deletions during fictive locomotion. The Journal of physiology 577(2):617-639

Schaal S (2006) Dynamic movement primitives-a framework for motor control in humans and humanoid robotics. In: Adaptive motion of animals and machines, Springer, pp 261-280

Seo K, Chung SJ, Slotine JJE (2010) Cpg-based control of a turtle-like underwater vehicle. Autonomous Robots 28(3):247-269

Shan J, Nagashima F (2002) Neural locomotion controller design and implementation for humanoid robot hoap-1. In: 20th annual conference of the robotics society of Japan

Sprowitz A, Pouya S, Bonardi S, Van Den Kieboom J, Mockel R, Billard A, Dillenbourg P, Ijspeert AJ (2010) Roombots: reconfigurable robots for adaptive furniture. IEEE Computational Intelligence Magazine 5(3):20-32

Taga G (1995) A model of the neuro-musculo-skeletal system for human locomotion. Biological cybernetics 73(2):97-111

Taga G, Yamaguchi Y, Shimizu H (1991) Self-organized control of bipedal locomotion by neural oscillators in unpredictable environment. Biological cybernetics 65(3):147-159

Tagne G, Hénaff P, Gregori N (2016) Measurement and analysis of physical parameters of the handshake between two persons according to simple social contexts. In: Intelligent Robots and Systems (IROS), 2016 IEEE/RSJ International Conference on, pp 674-679

Wang T, Hu Y, Liang J (2013) Learning to swim: a dynamical systems approach to mimicking fish swimming with cpg. Robotica 31(3):361-369

Williamson MM (1998) Rhythmic robot arm control using oscillators. In: Intelligent Robots and Systems, 1998. Proceedings., 1998 IEEE/RSJ International Conference on, IEEE, vol 1, pp 77-83

Wu X, Ma S (2010) Adaptive creeping locomotion of a cpg-controlled snake-like robot to environment change. Autonomous Robots 28(3):283-294

Xu W, Fang FC, Bronlund J, Potgieter J (2009) Generation of rhythmic and voluntary patterns of mastication using matsuoka oscillator for a humanoid chewing robot. Mechatronics 19(2):205-217

Yang W, Bae JH, Oh Y, Chong NY, You BJ, Oh SR (2010) Cpg based self-adapting multi-dof robotic arm 
control. In: Intelligent Robots and Systems (IROS), 2010 IEEE/RSJ International Conference on, IEEE, pp 4236-4243

Yu J, Tan M, Chen J, Zhang J (2014) A survey on cpginspired control models and system implementation. IEEE Transactions on neural networks and learning systems 25(3):441-456

Zehr EP, Carroll TJ, Chua R, Collins DF, Frigon A, Haridas C, Hundza SR, Thompson AK (2004) Possible contributions of cpg activity to the control of rhythmic human arm movement. Canadian journal of physiology and pharmacology 82(8-9):556-568

Zhou C, Low K (2012) Design and locomotion control of a biomimetic underwater vehicle with fin propulsion. IEEE/ASME Transactions on Mechatronics 17(1):25-35 